\title{
Propulsive Capability of an Asymmetric GDM Propulsion System
}

\author{
T. Kammash ${ }^{*}$ and R. Tang ${ }^{\dagger}$ \\ University of Michigan, Ann Arbor, MI, 48109
}

\begin{abstract}
The role of the ambipolar potential in the propulsive capability of the Gasdynamic Mirror (GDM) has been studied previously. The electrostatic potential arises as a result of the initial rapid escape of the electrons due to their small mass, leaving behind an excess of ions and creating a positive electric potential that slows down the electron escape while speeding up the ions until their respective axial diffusion are equalized. As a result, the ion confinement time is reduced while the ion escape energy is increased by an amount equal to the potential, translating into increased thrust and specific impulse. Previous studies, however, have only considered a GDM configuration with equal mirror ratios at both ends such that on average half of the ions escape through either end of the GDM. For a thruster it is desirable to bias the direction of ion escape to the thrusting end of the GDM. This can be achieved by properly controlling the mirror ratio at both ends such that on average a specified fraction of ions escape through the thrusting end. Due to this asymmetry, the magnitude of the ambipolar potential is changed, and as a result, the ion confinement time and escape energy are affected resulting in different propulsive capabilities compared to the case of a symmetric system.
\end{abstract}

\section{Nomenclature}

$\begin{array}{ll}A_{c} & =\text { area of plasma core } \\ A_{0} & =\text { mirror area } \\ D & =\text { axial diffusion coefficient } \\ \vec{E} & =\text { electric field } \\ E_{e} & =\text { electron energy } \\ E_{L} & =\text { escape energy } \\ e & =\text { electron charge } \\ k & =\text { density scale length } \\ L & =\text { length of plasma } \\ \ln \Lambda & =\text { Coulomb logarithm } \\ m & =\text { particle mass } \\ n & =\text { particle density } \\ R & =\text { plasma mirror ratio } \\ T & =\text { temperature } \\ Z & =\text { charge number } \\ v & =\text { monoenergetic particle velocity } \\ \gamma & =\text { monoenergetic particle flux } \\ \Gamma & =\text { velocity-averaged particle flux } \\ \mu & =\text { mobility } \\ \tau & =\text { confinement time } \\ \phi & =\text { electrostatic potential } \\ v & =\text { collision frequency }\end{array}$

\footnotetext{
* Professor Emeritus, Dept. of Nuclear Engineering and Radiological Sciences, 2355 Bonisteel Blvd, Ann Arbor, MI 48109, AIAA Associate Fellow.

${ }^{\dagger}$ Graduate Student, Dept. of Aerospace Engineering, 1320 Beal Ave, Ann Arbor, MI 48109. 


\section{Introduction}

$\mathrm{T}$

he gasdynamic mirror (GDM) fusion propulsion system is a magnetic mirror confinement system in which a hot dense plasma is confined long enough to allow fusion reactions to take place while allowing a fraction of the charged particles to escape to produce the desired thrust. The underlying confinement principle is based on the premise that the plasma density and temperature will have such values as to make the ion-ion collision mean free path much shorter than the plasma length. Under these conditions the plasma behaves like a fluid, and its escape from the system would be analogous to the flow of a gas into vacuum from a vessel with a hole. In our previous work, ${ }^{1}$ we assessed the propulsive capability of GDM by solving the appropriate particle and energy balance equations in order to establish the physical properties of the system as well as the required input energy and the reactor gain factor. The electrostatic potential that arises due to the rapid loss of the electrons was considered in later work, ${ }^{2}$ and its effect on the escape energies of the electrons and ions (hence the thrust and specific impulse of the system) was investigated. The GDM device could also be used as a plasma thruster if means other than fusion power are used to heat the plasma, e.g. radiative heating. In the latter case, the magnetic mirror confines the plasma during the heating process, after which it is ejected from the mirror end which serves as a magnetic nozzle. In both instances, sufficiently long confinement is needed, and the impact of the electrostatic potential on the confinement time as well as on the propulsive performance of the system is critical. In this paper we address the physics of such a potential and assess its effect on the escape energies of the electrons and ions irrespective of whether the GDM is fusion-powered or heated by other means.

\section{Mathematical Formulation}

In our previous analysis we computed the confinement time in GDM by calculating the particle flux through the mirrors and dividing it into the total number of particles in the system. Because of the initial rapid loss of the electrons due to their small mass, the particle dynamics is further influenced by the electric field associated with the electrostatic potential. Therefore, to properly address such dynamics, and to identify the forces that contribute to the flow of particles through the mirrors, one must incorporate not only the diffusion due to collisions, but also the contribution of the electric field. Our previous studies, however, assumed equal mirror ratios at both ends of the GDM, resulting in half of the particles on average escaping through either end of the GDM. For a thruster, it is desirable to bias the direction of particle escape to the thrusting end of the GDM. This can be achieved by properly controlling the mirror ratio at both ends.

\section{A. Ambipolar Potential and Loss Rate}

We begin with the monoenergetic diffusion equations for the electrons and ions in the device. ${ }^{3}$

$$
\begin{aligned}
& \left(1 / R_{j}\right) \gamma_{e j}=-D_{e} \nabla n_{e}-\mu_{e} \vec{E} n_{e} \\
& \left(1 / R_{j}\right) \gamma_{i j}=-D_{i j} \nabla n_{i}-\mu_{i} Z \vec{E} n_{i}
\end{aligned}
$$

where the mirror ratio reflects the fact that the monoenergetic flux $\gamma$ is measured at the throat of the mirror with area $A_{0}$. The subscript $j$ denotes the corresponding quantity at the two "mirrors". If the plasma area at the center of the device is $A_{c}$, then $A_{0}=A_{c} / R$. It is assumed that the ion and electron densities vary as

$$
n=n_{c} \exp [-(2 k / L) z]
$$

where $L$ is the axial length of the system and $k$ is an integer. It is clear that we can write for the total monoenergetic flux through the mirror the result

$$
A_{0} \gamma=-A_{0} D \nabla n=(2 k / L) A_{0} D n
$$

For the ions we employ the diffusion coefficient given by

$$
D_{i j}=\frac{L^{2}}{4 k \tau_{j}}
$$


where $\tau_{\mathrm{j}}$ is the loss time constant for mirror ratio $R_{j}$. The approximate loss time constant for ions $\tau_{\mathrm{ij}}$ is given by the Eq. (6). Note that this is based on flux balance and expresses the loss rate assuming all of the ions escape through one end of the GDM with mirror ratio $R_{j}$ in the absence of any electric field.

$$
\tau_{i j}=\frac{R_{j} L}{v_{t h}}
$$

For the electrons we use the following diffusion coefficient

$$
D_{e}=\frac{v_{e}^{2}}{3 v_{e i}}
$$

where $v_{e i}$ is the electron-ion collision frequency given by

$$
v_{e i}=\frac{n_{e} \ln \Lambda}{C_{0} E_{e}^{3 / 2}}
$$

with the constant $C_{0}=8.176 \times 10^{9} \mathrm{~s} \mathrm{~cm}^{-3} \mathrm{keV}^{-3 / 2}$. In addition we employ the following definitions for the mobilities appearing in Eqs. (1) and (2), namely

$$
\begin{aligned}
& \mu_{e}=\frac{e}{m_{e} v_{e i}} \\
& \mu_{i}=\frac{Z e}{m_{i} v_{e i}}
\end{aligned}
$$

Furthermore, assuming that the electrostatic potential varies in space in the same manner as the electron and ion densities, the electric field becomes

$$
\vec{E}=-\nabla \phi=(2 k / L) \phi
$$

With that the monoenergetic fluxes given by Eqs. (1) and (2) can be rewritten. Because the plasma in GDM is highly collisional, it is reasonable to assume that the species have Maxwell-Boltzmann velocity distributions from which the total electron and ion fluxes $\Gamma_{e j}$ and $\Gamma_{i j}$ can be found. ${ }^{2}$ The condition of charge neutrality requires that the charged flux losses be equal and the net charge be zero, i.e.

$$
\begin{aligned}
& \Gamma_{j} \equiv \Gamma_{e j}=Z \Gamma_{i j} \\
& n \equiv n_{e}=Z n_{i}
\end{aligned}
$$

Upon satisfying these conditions and defining the following quantities, Eq. (13), the following balance equation is obtained for the electrostatic potential $\phi$, namely Eq. (14a).

$$
\begin{aligned}
& \delta_{j} \equiv \frac{L}{4 R_{j} k} m_{e} v_{e i}\left(\frac{8 T_{i}}{\pi m_{i}}\right)^{1 / 2} \\
& x \equiv \sqrt{\frac{3 e \phi}{2 T_{e}}} \\
&\left(1-\frac{2}{3} x^{2}\right)[1-\operatorname{erf}(x)]+\frac{2}{\sqrt{\pi}} x e^{-x^{2}}=\frac{\delta_{j}}{T_{e}}+\frac{2}{3} \frac{m_{e}}{m_{i}} Z^{2} x^{2}
\end{aligned}
$$


where $T_{e}$ and $T_{i}$ are the electron and ion temperatures respectively and $\operatorname{erf}(x)=(2 / \sqrt{\pi}) \int_{0}^{x} e^{-t^{2}} d t$ is the error function.

We recall that Eq. (14a) corresponds to two equations for the two "mirrors", one for $j=1$ and one for $j=2$, and in general, the potentials that satisfy the two are different. However, unlike fluxes, we do not have two distinct potentials inside the GDM. As a result, the two equations resulting from Eq. (14a) are combined into Eq. (14b), which is then used to determine the ambipolar potential inside the GDM.

$$
\left(1-\frac{2}{3} x^{2}\right)[1-e r f(x)]+\frac{2}{\sqrt{\pi}} x e^{-x^{2}}=\frac{\delta_{1}+\delta_{2}}{2 T_{e}}+\frac{2}{3} \frac{m_{e}}{m_{i}} Z^{2} x^{2}
$$

Returning to Eq. (5), we can express the loss time constant as

$$
\tau_{j}=\frac{L^{2}}{4 k D_{i j}}
$$

where the diffusion coefficient $D_{i j}$ is given by Eq. (16) and is obtained from the condition $\left(1 / R_{j}\right) \Gamma_{j}=-D_{i j} \nabla n=(2 k / L) D_{i j} n$, together with the expression for the total ion flux ${ }^{2}$ and Eq. (12), i.e.

$$
D_{i j}=\frac{1}{m_{e} v_{e i}}\left[\delta_{j}+\frac{m_{e}}{m_{i}} Z^{2} e \phi\right]
$$

Writing the following for the ion thermal velocity,

$$
v_{t h}=\sqrt{\frac{8 T_{i}}{\pi m_{i}}}
$$

and using this to rewrite $\delta_{j}$ in terms of $\tau_{i j}$ in Eq. (16), and upon equating the resulting expression for the ion diffusion coefficient to Eq. (5), we obtain the loss time constant, or equivalently the confinement time, namely

$$
\tau_{j}=\frac{\tau_{i j}}{1+\left(m_{e} / m_{i}\right) Z^{2}\left(e \phi / \delta_{j}\right)}
$$

We observe that when the potential $\phi$ is neglected, the characteristic confinement time reduces to that given by Eq. (6), which was used in the previous studies of GDM. ${ }^{1}$ Again as before, Eq. (18) expresses the loss rate assuming that all the ions escape through one end of the GDM with mirror ratio $R_{j}$. The total loss rate can be written as follows.

$$
\frac{1}{\tau}=\frac{1}{\tau_{1}}+\frac{1}{\tau_{2}}
$$

When the two mirror ratios are the same, Eq. (19) reduces to the expression given in Ref. 2, with the caveat that the total potential-less loss rate is now given by $\tau_{i}=(R L) /\left(2 v_{t h}\right)$, which has an additional factor of $1 / 2$ compared to Ref. 2. In addition to preserving consistency, this additional factor also arises naturally from flux balance consideration.

\section{B. Average Particle Escape Energy}

It should be noted in Eq. (14) that the mirror ratio $R$ appears only in the ion dynamics term since only the ions respond to mirror confinement as noted earlier. Moreover, $T_{e} \neq T_{i}$ in GDM since the electron distribution is in effect a "truncated" Maxwellian because electrons with energies above that of the potential do escape. The potential is 
obtained from Eq. (14) when solved iteratively, and it can be used to evaluate the electron and ion escape energies, which can be expressed by

$$
\begin{gathered}
E_{L e}=\frac{\left(5-2 x^{2}\right)[1-e r f(x)]+\frac{2}{\sqrt{\pi}}\left(5+\frac{4}{3} x^{2}\right) x e^{-x^{2}}}{2\left(1-\frac{2}{3} x^{2}\right)[1-e r f(x)]+\frac{4}{\sqrt{\pi}} x e^{-x^{2}}} T_{e} \\
E_{L i j}=\frac{2+\frac{m_{e}}{m_{i}} Z^{2} \frac{T_{e}}{\delta_{j}} x^{2}}{1+\frac{2}{3} \frac{m_{e}}{m_{i}} Z^{2} \frac{T_{e}}{\delta_{j}} x^{2}} T_{i}
\end{gathered}
$$

We note that the average electron escape energy for asymmetric mirrors, Eq. (20), is the same as in the case for symmetric mirrors. Again, this is expected since the electrons have such small mass and high velocity that they essentially do not see the mirrors. On the other hand, the average ion escape energy has the same expression as that for symmetric mirrors, but due to its dependence on the mirror ratio, the escape energy will be different at both ends of an asymmetric GDM. Also note that if the potential is ignored, the electron and ion escape energies reduce to

$$
E_{L e}=\frac{5}{2} T_{e}, \quad E_{L i j}=2 T_{i}
$$

which agree with previous results. ${ }^{1}$ Furthermore, since $E_{L e}$ and $E_{L i j}$ are the average energies of escaping electrons and ions as they leave the plasma chamber, the ambipolar potential must be added (subtracted) to the ion (electron) energy to obtain their energies outside the chamber. Therefore, the average energy of an escaped electron outside the plasma chamber is $\left(E_{L e}-e \phi\right)$ and that of an escaped ion is $\left(E_{L i j}+e \phi\right)$.

\section{Conclusion}

We have developed the mathematical analysis that predicts the effect of the electrostatic potential on the flow of the propellant in the gasdynamic mirror propulsion system and extended it to the case of an asymmetric system. We have explicitly demonstrated the role of such a potential in the escape energies of both the electrons and the ions of the system, which in turn dictate the values of the specific impulse and thrust produced by the GDM thruster. For propulsion applications it is desirable to bias the direction of ion escape to the thrusting end of the GDM, which can be achieved by properly controlling the magnetic field strengths at the two ends. As a result, the thrust and specific impulse will increase. However, the confinement time for the asymmetric device may also increase compared to the symmetric device, leading to an increase in the length and mass of the system. On the other hand, the longer confinement time will allow for longer heating time for the ions. This in turn will make the choice of the heating scheme more flexible.

\section{References}

${ }^{1}$ Kammash, T., and Lee, M. J., “Gasdynamic Fusion Propulsion System for Space Exploration,” Journal of Propulsion and Power, Vol. 11, No. 3, 1995, pp. 544-553.

${ }^{2}$ Kammash, T., and Galbraith, D. L., "Improved Physics Model for the Gasdynamic Mirror Fusion Propulsion System," Journal of Propulsion and Power, Vol. 14, No. 1, 1998, pp. 24-28.

${ }^{3}$ Rose, D. J., and Clark, M., Plasma and Controlled Fusion, Wiley, New York, 1961, pp. 66. 